\title{
Very faint X-ray transients in our Galaxy
}

\author{
Rudy Wijnands ${ }^{1}$ \\ ${ }^{1}$ Astronomical Institute "Anton Pannekoek", University of Amsterdam, Kruislaan 403, \\ 1098 SJ, Amsterdam, The Netherlands \\ email: rudy@science.uva.nl
}

\begin{abstract}
We present the first results of our X-ray monitoring campaign using the X-ray satellites XMM-Newton and Chandra on the 1.7 square degree region centered on Sgr A*. The purpose of this campaign is to monitor the X-ray behavior of X-ray sources (both persistent and transient ones) which are too faint to be detected by monitoring instruments aboard other satellites currently in orbit (e.g., RXTE, Integral, Swift). Our first observations were obtained on June 5, 2005, using the HRC-I detector aboard Chandra. Here we briefly discuss our results on the very faint X-ray transients we detected during these observations. We detected new outbursts (with peak luminosities close to $10^{36} \mathrm{erg} \mathrm{s}^{-1}$ ) of the two very faint transients GRS 1741.9-2853 and XMM J174457-2850.3, as well as a potential new very faint X-ray transient with an outburst luminosity of a few times $10^{34} \mathrm{erg} \mathrm{s}^{-1}$.
\end{abstract}

Keywords. accretion, accretion disks, X-rays: binaries, stars: neutron

\section{Introduction}

The X-ray luminosity of our Galaxy is dominated by accreting neutron stars and black holes in X-ray binaries. The majority of such systems is usually dormant but occasionally they exhibit outbursts during which their X-ray luminosity increases by a factor of $>100$ with peak luminosities of $>10^{37-39} \mathrm{ergs} \mathrm{s}^{-1}$. The existence of these bright $X$-ray transients, mostly containing black holes, has been known since the early days of X-ray astronomy. Several years ago, Heise et al. (1999), using the BeppoSAX satellite, provided strong evidence for a class of faint $X$-ray transients with peak luminosities of of $10^{36-37} \mathrm{ergs} \mathrm{s}^{-1}$. Interestingly, a large fraction of these faint systems harbor neutron stars instead of black holes, pointing to differences in formation histories (King(2000)).

Although transients with peak luminosities below $10^{36} \mathrm{erg} \mathrm{s}^{-1}$ have been sporadically reported in the past (e.g., Maeda et al. (1996)), it was only recently realized (e.g., Sakano et al. (2005)) that such very faint X-ray transients (VFXTs) likely form another distinct class of X-ray transients with peak luminosities of $10^{34-36} \mathrm{ergs} \mathrm{s}^{-1}$, a factor $>10$ lower than the faint transients. All recently discovered VFXTs were found using the sensitive Chandra and XMM-Newton X-ray satellites (Sidoli \& Mereghetti (2003), Hands et al. (2004), Porquet et al. (2005), Sakano et al. (2005), Muno et al. (2005a)). Some of them might be intrinsically bright transients at large distances; however, many are observed near the Galactic center, indicating source distances of $\sim 8 \mathrm{kpc}$ and therefore very low peak luminosities. Some VFXTs might be intrinsically brighter than observed due to inclination effects (e.g., Muno et al. (2005b)), but only a small fraction of the VFXTs can be explained in this manner (see, e.g., the discussion in King \& Wijnands (2005)).

The characteristics of the VFXTs (e.g., their spectra or outburst light curves; e.g., Sakano et al. (2005) and Muno et al. (2005a)) indicate that they are not a homogeneous class of sources but that different types of accreting neutron stars and black holes show themselves as VFXTs. Therefore, a variety of models are probably needed to explain all systems. However, it is likely that a significant fraction of the VFXTs are neutron stars 


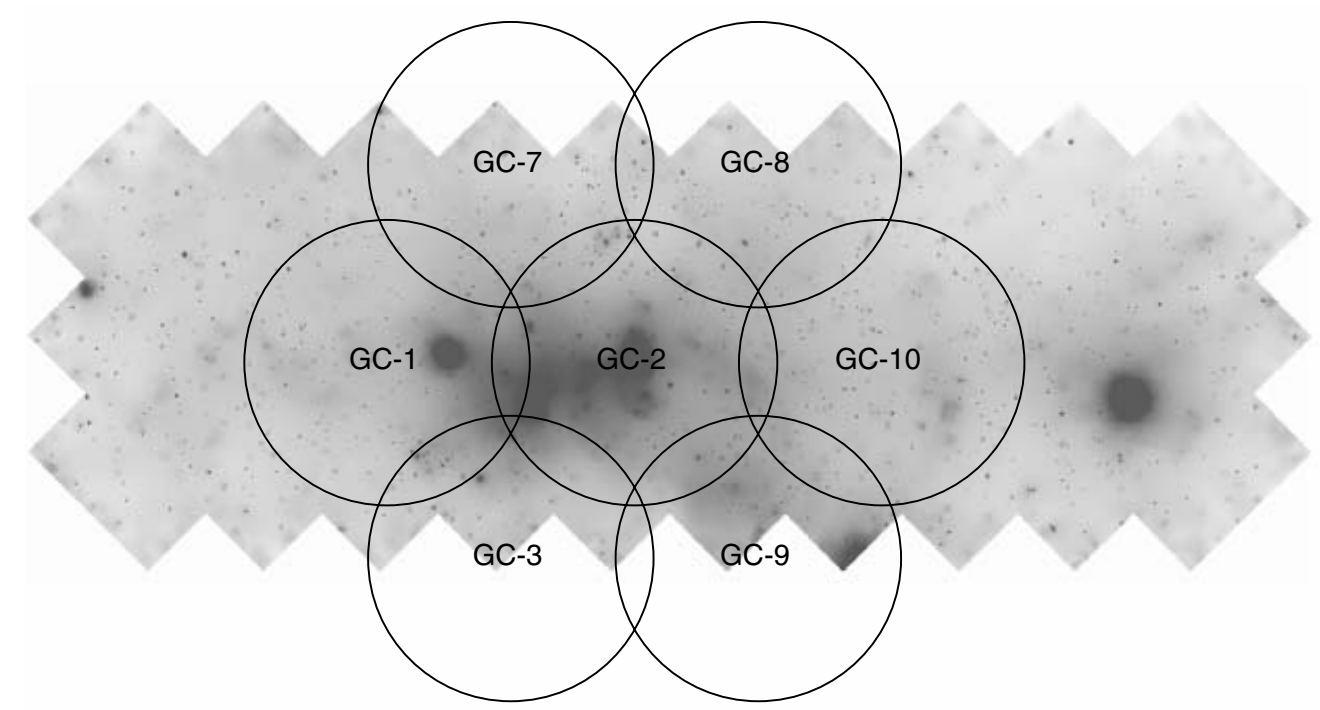

Figure 1. Field-of-view of the monitoring campaign in Galactic coordinates. The circles indicate the field-of-view of the Chandra and XMM-Newton observations. The image is the Wang et al. (2002) Chandra Galactic center survey data.

and black holes which accrete matter at a very low rate from a low-mass companion star. For example, at least two such systems have exhibited type-I bursts which have so far only been observed for accreting neutron stars in low-mass X-ray binaries. These lowmass X-ray binaries which accrete at very low rates are a challenge to our understanding of the evolution of such systems. If their time averaged accretion rates do not drop below $10^{-13} M_{\odot} \mathrm{yr}^{-1}$ then these systems can be explained as low-mass X-ray binaries which have spent most of the age of the Galaxy reducing their companion stars to values $\sim 0.01 M_{\odot}($ King \& Wijnands (2005)). If future observations show that the time averaged accretion rates drop below this value then more exotic explanations may be needed, such as accretion from planetary companions or accretion onto intermediate mass black holes.

Our understanding of the nature of these enigmatic VFXTs is hampered by the lack of known systems, the limited information about their outburst duration, recurrence time and duty cycle (e.g., Muno et al. (2005a)), and the lack of optical/infrared counterparts. The VFXTs are usually too faint (if close to the Galactic center) to be detected by the current monitoring satellites (e.g., RXTE, Integral or Swift) and source confusion close to the Galactic center also inhibits their detection. Therefore, we have initiated a monitoring campaign using XMM-Newton and Chandra of the Galactic center region to discover new VFXTs and to detect new outbursts of the already known VFXTs.

\section{Description of the program}

We have secured a monitoring campaign using XMM-Newton and Chandra of the 1.7 square degree region centered around Sgr $A^{*}$ to search for new and recurrent VFXTs (Fig. 1). Our campaign consists of four observational epochs, two with Chandra (one performed on June 5 and the other scheduled for the week of 17 October 2005; using the HRC-I detector because of its relatively large field-of-view) and two with XMM-Newton (scheduled for the end of February and early April 2006). The first Chandra/HRC-I observations have been performed and we discuss our first results here. 

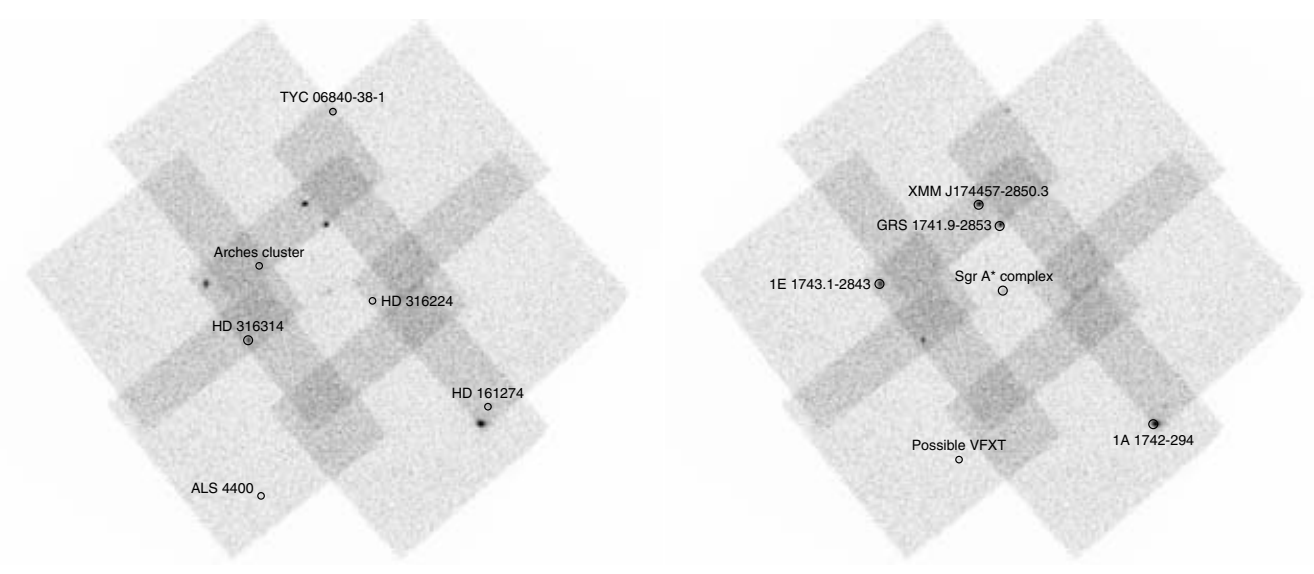

Figure 2. The merged image of the 7 HRC-I observations. Left panel: a sub-set of the detected stars is indicated; right panel: the detected X-ray binaries as well as the Sgr A* region.

\section{Results}

The combined image of the seven Chandra/HRC-I observations is shown in Figure 2. Preliminary searches for point sources resulted in 21 detected sources. Two of these sources (the Sgr A* region and the Arches cluster) are known to consist of a complex of point sources in combination with strong diffuse emission. Sgr A* itself was not detected during our observations. The majority of the remaining sources can be identified with known stars (e.g., HD 316314, HD 316224, TYC 06840-38-1) or have clear counterparts in the DSS images. Also, two persistent X-ray binaries (1E 1743.1-2843 and 1A 1742-294) are detected. Here we will not discuss those stars and persistent X-ray binaries; instead we will focus on the VFXTs detected.

Two known VFXTs were found to be in outburst again during our observations: GRS 1741.9-2853 and XMM J174457-2850.3 (Fig. 2 right panel; Wijnands et al. (2005)). Their luminosities are somewhat uncertain since the HRC-I detector does not have energy resolution. Therefore, we converted the observed count rate into luminosity using PIMMS and the spectra previously found for these sources (e.g., Muno et al. (2003), Sakano et al. (2005)) and found that both sources have luminosities slightly below $10^{36} \mathrm{erg} \mathrm{s}^{-1}$. No type-I bursts were seen for either source. After the discovery of the new outbursts of both sources we obtained an additional Chandra observation (using the ACIS-I detector) of the two sources on July 1, 2005. During this observation, we could detect GRS 1741.92853 still at a luminosity of $\sim 10^{35} \mathrm{erg} \mathrm{s}^{-1}$ but XMM J174457-2850.3 had decreased in luminosity to a few times $10^{33} \mathrm{erg} \mathrm{s}^{-1}$. The long term light curves of both sources are shown in Figure 3. A possible new VFXT (Fig. 2) was also detected at a luminosity of a few times $10^{34} \mathrm{erg} \mathrm{s}^{-1}$, which was not seen in archival XMM-Newton data.

\section{Conclusions}

During the first Chandra observations of our monitoring campaign we detected new outbursts of the known VFXTs GRS 1741.9-2853 and XMM J174457-2850.3. We also discovered a potential new VFXT at a peak luminosity of a few times $10^{34} \mathrm{erg} \mathrm{s}^{-1}$. Using our scheduled monitoring observations in the fall of 2005 and the spring of 2006, we will discover several more VFXTs and additional outbursts of known VFXTs. By studying the properties and behavior of many VFXTs we will get a better understanding of the nature of this enigmatic class of X-ray transients. For the systems accreting from a low-mass 

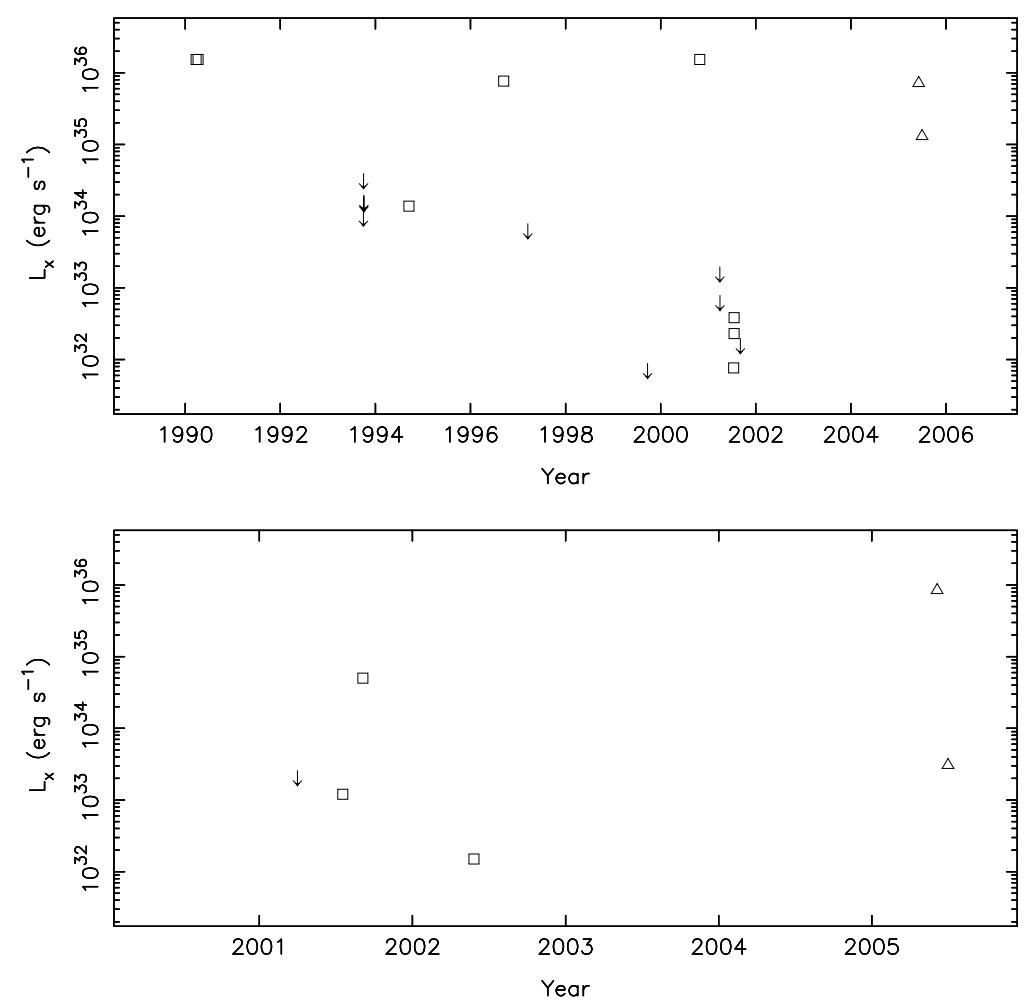

Figure 3. Light curves of GRS 1741.9-2853 (top) and XMM J174457-2850.3 (bottom). In both panels the triangles indicate our Chandra data. The squares and the upper limits are taken from, respectively, Muno et al. (2003) and Sakano et al. (2005) for the top and bottom panel.

companion star, our monitoring campaign will help to determine their time averaged accretion rates, needed to constrain theories about the formation of such systems (e.g., King \& Wijnands (2005)).

\section{References}

Hands, A. D. P., Warwick, R. S., Watson, M. G., \& Helfand, D. J. 2004, MNRAS, 351, 31

Heise, J., in’t Zand, M. J. J., Smith, S. M. J., Muller, M. J., Ubertini, P., Bazzano, A., Cocchi, M., \& Natalucci, L. 1999, Astrophysical Letters Communications, 38, 297

King, A. R. 2000, MNRAS, 315, L33

King, A.R. \& Wijnands, R. 2005, MNRAS, submitted

Maeda, Y., Koyama, K., Sakano, M., Takeshima, T., \& Yamauchi, S. 1996, PASJ, 48, 417

Muno, M. P., Baganoff, F. K., \& Arabadjis, J. S. 2003, ApJ, 598, 474

Muno, M. P., Pfahl, E., Baganoff, F. K., Brandt, W. N., Ghez, A., Lu, J., \& Morris, M. R. 2005a, ApJ, 622, L113

Muno, M.P., Lu, J.R., Baganoff, F. K., Brandt, W.N., Garmire, G.P., Ghez, A.M., Hornstein, S.D., \& Morris, M.R., 2005b, ApJ, submitted (astro-ph/0503572)

Porquet, D., Grosso, N., Burwitz, V., Andronov, I. L., Aschenbach, B., Predehl, P., \& Warwick, R. S. 2005, A\&A, 430, L9

Sakano, M., Warwick, R. S., Decourchelle, A., \& Wang, Q. D. 2005, MNRAS, 357, 1211

Sidoli, L., \& Mereghetti, S. 2003, The Astronomer's Telegram, 147, 1

Wang, Q. D., Gotthelf, E. V., \& Lang, C. C. 2002, Nature, 415, 148

Wijnands, R., et al. 2005, The Astronomer's Telegram, 512, 1 


\section{Discussion}

IVANOVA: What can you say about outburst's durations and recurrence intervals for VFXT?

WiJNANDS: Both the durations and recurrence times vary a lot from source to source. Some VFXTs are on for $<1$ month, others $>1$ year. Not much is known about the recurrence time but at least a few recur every 3-5 years.

Chernyakova: What is the impact of the newly resolved point sources in the Sgr A* region to the total X-ray emission of the region?

Wijnands: Close $\left(<10^{\prime}\right)$ to Sgr $\mathrm{A}^{*}$ the X-ray flux is consistent with what has been found before (e.g. by Muno et al). In the whole FOV of our MRC-I observations the true VFXTs detected contribute about $210^{36} \mathrm{erg} / \mathrm{s}(2-10 \mathrm{KeV})$ to the X-ray luminosity in this region.

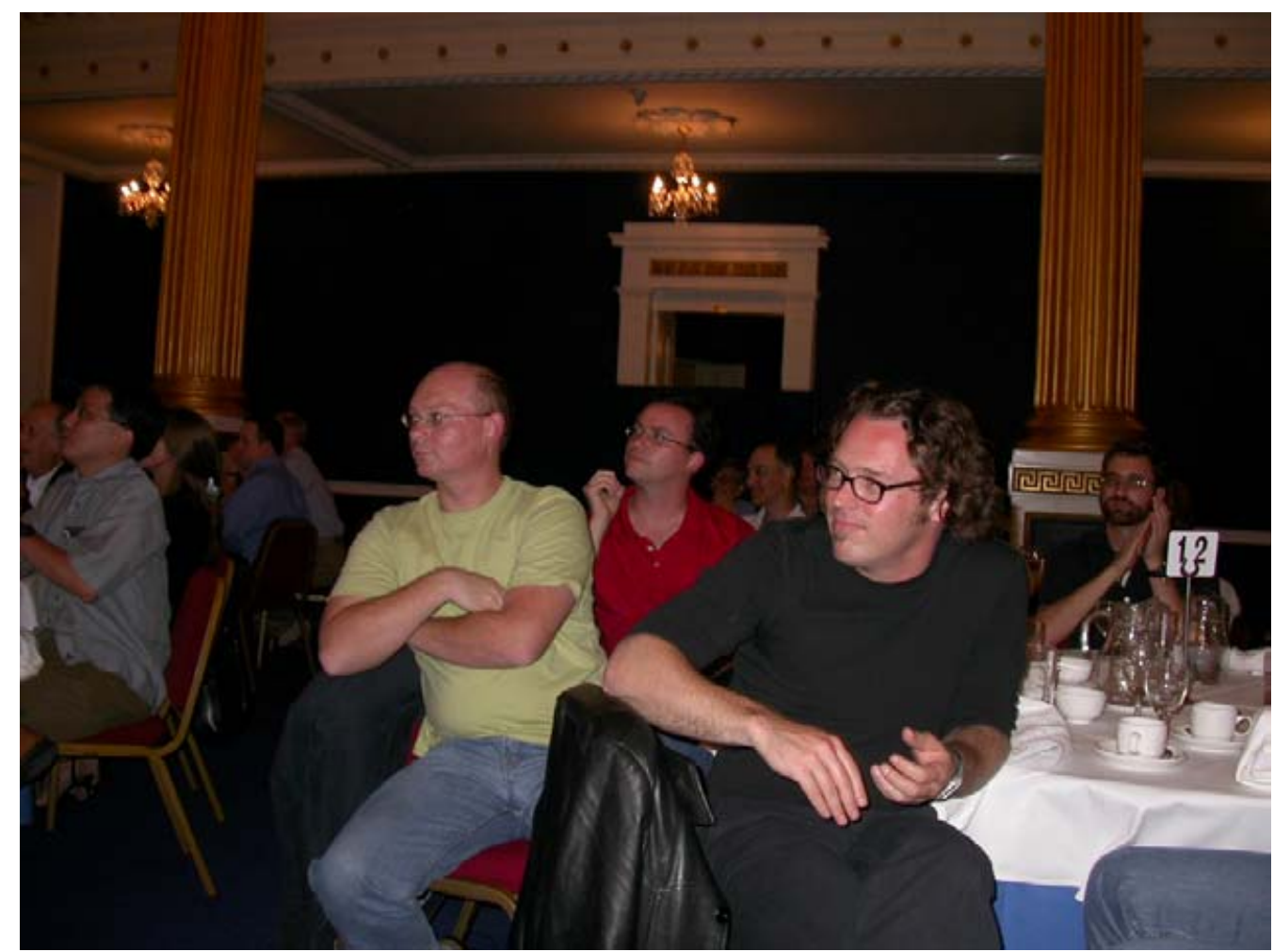

Rudy Wijnands, Tom Maccarone and Jacco Vink (from left to right) follow eagerly the demonstration of modern Irish dancing. 